\title{
Contrôle ou libération \\ des émotions dans le contexte \\ des pratiques de santé qigong
}

\section{Evelyne Micollier}

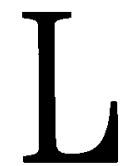

E qigong contemporain, « exercices de l'énergie vitale ", "pratiques du $q i »$, ou encore «travail du $q i$ », est un ensemble de techniques corporelles à fins thérapeutiques qui s'enracinent dans la tradition chinoise: $q i$ peut être traduit par " souffle, énergie, énergie vitale circulant dans le corps en correspondance avec des énergies cosmiques, force, force vitale, influences, air, substance "; gong signifie " exercice, pratique, travail, talent artistique, mérite, qualité, technique, art, temps ». Les associations de sens des deux caractères $q i$ et gong sont choisies selon le contexte. Ces pratiques sont d'ordre «psychophysiologique " pour donner une traduction du point de vue des catégories occidentales. Elles visent à l'entretien de la santé, au prolongement de la vie humaine et à la régression des états pathologiques. Les principes de base sont l'exercice de la relaxation (fangsong), de la respiration (huxi [inspirer, expirer]), du rejet et de l'assimilation $(\text { tuina })^{(1)}$ et de la pensée yinian ${ }^{(2)}$. Des pratiques de santé qui mettent l'emphase sur des techniques précises de respiration ne sont en rien originales ; en revanche, l'utilisation dans la pratique du concept yi défini comme un ensemble d'élaborations mentales conscientes incluant des idées, des émotions, des pensées, des connaissances intuitives et des produits de l'imagination, est spécifique au qigong qui devient alors un exercice de l'esprit. Une pratique approfondie aboutit à une véritable transformation de l'être par des étapes successives franchies grâce à un travail régulier du $q i$ : l'acquisition de l'état de santé, sa préservation et l'absence de maladie sont les premiers résultats de l'entraînement et déjà non des moindres si l'on considère leur impact sur la vie. Ils conduiront graduellement à une longévité relative et au développement de la personne si le pratiquant persévère ${ }^{(3)}$.

Le qigong présente une autre originalité non plus dans le domaine des techniques mais par sa dimension sociale et les enjeux socio-politiques dont il est l'objet : offrant une lecture pertinente de la société chinoise contemporaine, il peut être considéré comme « une expression de la modernité chinoise ", selon le titre d'un article de C. Despeux ${ }^{(4)}$, comme un lieu où les contradictions locales s'expriment et se négocient. Un phénomène social aux multiples facettes s'est développé, en Chine, autour de ces pratiques par ses formes traditionnelles ou néo-traditionnelles aux innombrables variantes et, en Occident, par ses formes " exportées », « réinterpretées » ou complètement « inventées ». En Chine, le dynamisme de ce phénomène peut être mesuré par la diversité des formes de qigong, le développement de formes d'organisations sociales centrées sur les activités liées au qigong, le nombre de publications (ouvrages, matériaux éducatifs, revues) spécialisées, universitaires ou populaires ${ }^{(5)}$. Des citadins appartenant à toutes les catégories sociales manifestent un intérêt croissant pour le qigong depuis une quinzaine d'années. 
A l'échelle nationale, plusieurs milliers de formes de $q i$ gong, des centaines d'associations et une dizaine de revues spécialisées ont vu le jour. Selon des estimations officielles, 50 millions de personnes étaient engagées dans des activités relatives au qigong au début des années $1990^{(6)}$. Parmi elles, 20 millions seraient membres d'organisations sociales associatives officielles, semi-officielles ou populaires. Les données officielles doivent bien sûr être manipulées avec précaution dans le contexte chinois. Il y a dix ans, les pratiquants du qigong étaient probablement déjà beaucoup plus nombreux et il semble que leur nombre semble n'a cessé d'augmenter depuis. Il suffit de rappeler les événements récents qui ont animé le centre de Pékin pour mesurer l'ampleur et les enjeux socio-politiques du phénomène : plus de dix milles adeptes du Falungong, une forme de qigong bouddhique, assis dans la position du « lotus », ont manifesté devant le siège du gouvernement pour réclamer la reconnaissance de leur groupe et la levée de l'interdiction sanctionnant la publication la plus récente de leur maître et fondateur du mouvement, Li Hongzhi. Cette manifestation pacifique et bien organisée est le plus grand rassemblement contestataire dans la capitale chinoise depuis le printemps de Pékin de $1989^{(7)}$. Selon des estimations officielles, les adeptes de ce mouvement en Chine seraient plus nombreux que les 55 millions de membres du Parti communiste chinois... (Cf. l'article cijoint de B. Vermander.)

La méthodologie qui sous-tend cette recherche s'inscrit dans la discipline ethnologique. Les données ethnographiques ont été recueillies par l'observation participante, l'analyse des discours à partir d'un corpus d'entretiens non-directifs ou semi-directifs de recherche enregistrés et la méthode biographique. Cette dernière méthode qui consiste à construire des récits de vie, suppose l'instauration de relations privilégiées avec l'informateur établies par des entrevues fréquentes, durables, et des entretiens approfondis. Les enquêtes sur les pratiques de qigong envisagées dans leurs dimensions thérapeutique et sociale ont été effectuées à Canton en 1991-1992 (7 mois) et à Taipei en 1995 (7 mois). La revue terminologique s'appuie sur des données issues d'entretiens enregistrés recueillis à Canton auprès de pratiquants de qigong exclusivement et à Taiwan auprès de pratiquants ou de non-pratiquants.

Lobjectif de cet article est de montrer que la manipulation des émotions par les différents acteurs sociaux en présence - le maître, le groupe et l'individu - est une technique de soins dans le contexte des pratiques de qigong. La première partie porte sur le vocabulaire des émotions étudié à partir de trois notions clés qui aident à comprendre le processus de conceptualisation des émotions dans le contexte culturel chinois : (1) $q i$ «force vitale », (2) shen « corps-personne » et (3) ganjue, ganqing, jidong, trois termes désignant " émotion". La seconde partie aborde la sociabilité et la subjectivité des émotions à partir de l'analyse des conduites des pratiquants de qigong. La troisième partie centre son attention sur l'un des aspects de la pratique - les états de transe générés par les exercices dans certaines conditions - parce qu'ils apparaissent comme l'expression subjective et sociale d'émotions contrôlées ou libérées.

\section{Revue terminologique}

Deux aspects du langage justifient l'intérêt d'une revue terminologique : le langage véhicule la vision du monde des personnes qui l'utilisent; les usagers ne sont pas conscients de sa signification implicite. La pertinence du contexte pour saisir le sens précis des termes ou des expressions est un autre trait, particulièrement significatif lorsqu'il est appliqué à la langue chinoise. Pour souligner la difficulté de la traduction et expliquer les critères de choix d'un sens au détriment d'un autre, précisons que la langue chinoise est fortement polysémique. Le traducteur fait une démarche de contextualisation qui consiste à considérer l'ensemble des connotations, et il est encore parfois confronté à un choix difficile : dans ce cas, le sens le moins précis et le plus « polysémique » en français est préféré par l'auteur pour restituer cet aspect de la langue chinoise.

Les explications des termes qui signifient "émotion" ont été recueillies auprès d'informateurs taiwanais exclusivement, pratiquants ou non-pratiquants de qigong, de niveaux non homogènes d'éducation. Léchantillon est de vingt personnes. L'objet de recherche lié à la perception du corps et à l'expression des émotions n'était pas dévoilé aux personnes qui ont collaboré : des aspects de la vie quotidienne ont été abordés par les thèmes de la famille et du travail parce qu'ils mettent en jeu des valeurs fondamentales et peuvent être une voie d'accès à l'intimité des personnes. Une conversation sur la famille en particulier portant sur la filiation, les ascendants et les descendants, permet d'évoquer de manière indirecte la maladie, la vie et la mort.

Le qi est un concept de base de la médecine chinoise traditionnelle et, dans une perspective plus large, de la conceptualisation de la vie. D'un point de vue distinc- 


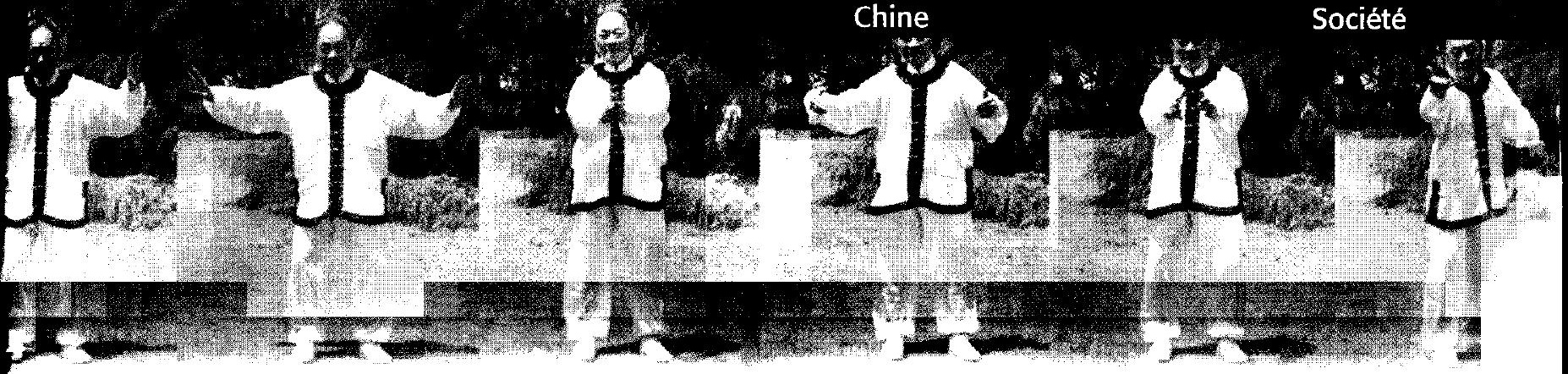

Les expressions idiomatiques en quatre caractères indiquent un seul sens générique, le corps-personne.

Le corps $t i$ apparait dans le bissyllabique shenti :

L'entrée nominale fait référence à trois sens portant sur la constitution physique du corps : (1) corps, partie du corps, (2) substance, (3) style, forme.

$\mathrm{Au}$ terme verbal, la notion de personne réapparait : (1) faire personnellement l'expérience de quelque chose, (2) se mettre soi-même dans la position d'autrui.

Les deux sens génériques de "constitution physique " et " d'expérience de la personne " sont présents de manière équivalente dans les bissyllabiques construits à partir de $t i$.

Les trois termes ganjue, ganqing, jidong (sentiment, passion, émotion) sont utilisés dans le sens d'émotion : les nuances de sens sont mentionnées dans la mesure du possible. Ganjue est employé pour désigner des sensations, des processus qui se produisent dans le corps : le terme est associé à la notion de "corps» shenti. Dans un dictionnaire usuel, ganjue a les sens de «sensation " et de "perception ». Ganqing signifie «sentiment " et éventuellement « émotion » quand le sentiment suscite des émotions. L'émotion est donc conçue comme l'expression d'un sentiment. Ganqing est associé facilement à ce qui se produit dans le cœur (xinli). L'analyse de contenu des discours met en évidence trois champs sémantiques :

(1) Le cour est le siège des états affectifs : xinqing (littéralement : " le sentiment du cœur ») signifie "l'humeur, l'état d'esprit "; xinli tou hen nanguo (littéralement : «dans le coeur, très triste ») indique que la personne est très triste. En chinois, la plupart des caractères qui désignent des états émotionnels contiennent dans la graphie le radical «cœeur » comme $s i$, bei, kong. Le caractère si comporte deux sens : l'un, " contemplation ", désigne l'une des techniques qui conduit au développement de la personne dans les pratiques de qigong et est connoté positivement ; l'autre réfère à un état pathologique, "inquiétude, mélancolie " et contient une connotation négative. Les émotions sont alors considérées comme les symptômes d'une maladie.

(2) Le concept de cœur a une connotation morale.
L'expression xin li bu hao (littéralement: «dans le cour, ce n'est pas bon, ce n'est pas bien ") indique que la personne n'est pas recommandable.

(3) Le cœur est le siège de l'esprit. L'acte de penser est associé au cœur et non au cerveau comme le montre par exemple l'expression shenxin jiankang (sain de corps et d'esprit).

Le cœur est rattaché comme le corps à la notion de personne en y ajoutant une connotation morale et une fonction de contrôle de la pensée et donc des actes commandés par cette pensée. Par rapport au qi qui donne la vie, le cœur la régule et la gouverne. Dans la tradition, le cour est le siège de l'esprit, des émotions et des sentiments. Les représentations qui sous-tendent les discours des informateurs sont en cohérence avec le concept traditionnel. Un dictionnaire usuel indique deux sens du terme ganqing (1) sentiment, émotion, (2) affection, amour ${ }^{(1)}$.

Le troisième terme désignant l'émotion est jidong associé plus précisément à une expression très visible parfois violente des états émotionnels. Cette manière démonstrative d'exprimer les émotions suggère des transformations du corps et de la personne shenti bianhua. Jidong sert à qualifier des émotions liées à la passion (jiqing) ${ }^{(12)}$. Dans un dictionnaire usuel, le monosyllabique dong a le sens de " mouvement » ou « agitation ». Le verbe jidong signifie « exciter, stimuler, agiter, remuer ». Le monosyllabique $j i$ porte les sens de « surgir, stimuler » et en tant qu'adjectif ceux de « violent, féroce et aigu ».

\section{Approche des émotions dans la pratique}

Si l'objectif du pratiquant est l'entretien de la santé, le contrôle des émotions est nécessaire pour être à l'écoute des processus qui se produisent dans le corps. Ils sont vécus du point de vue distinctif comme corporels. Le qi doit être guidé par la pensée (yong yinian) dans la pratique individuelle ou collective. Dans ce contexte, il constitue l'élément vital, dans le sens littéral de "donner vie ", circulant dans la personne selon des trajets précis en correspondance avec des énergies cosmiques : la personne ne doit alors être réceptive 
qu'aux processus qui se produisent à l'intérieur de la personne ; aucun processus d'interaction entre les pratiquants n'est visible. Si l'objectif est la thérapie par $q i$ gong, le $q i$ bénéfique du maître est envoyé dans le corps du patient. Cette technique de traitement à distance (faqi, fagong: " envoyer le $q i »$ ), fait partie des constantes du traitement par qigong. Libérer les émotions est un comportement nécessaire pour être réceptif au $q i$ du maître : les pratiquants doivent manifester leurs états émotionnels sans contrôle et sans inhibition. Soyons à l'écoute de Maître $\mathrm{X}^{(13)}$ lors d'une séance de traitement collectif par projection de $q i$ : « Décontractez-vous. Si vous avez envie de crier, criez ; si vous avez envie de rire, riez; si vous êtes tristes, vous avez envie de pleurer, pleurez ; de danser, dansez; de bouger, de faire des mouvements, faites-le ".

Si l'action du $q i$ du maitre dans le corps du patient a des effets indésirables qui peuvent être détectés par des expressions émotionnelles "désordonnées " (luandong) ${ }^{(14)}$, de l'avis du maître, c'est lui qui doit contrôler les débordements. La pratique est souvent collective car, comme dit le maître, son propre $q i$ est plus efficace s'il entre en contact avec le $q i$ de chaque personne dans le groupe. Les effets de $q i$ s'ajoutent les uns aux autres. Dans ce contexte, la communication entre les participants est visible. Le " patient-pratiquant " doit être capable, selon le contexte et sa motivation, de contrôler ou de libérer les émotions. D'une part, il exprime ce qu'il ressent dans un processus d'interaction avec l'extérieur. En pratiquant ensemble, une relation se noue entre la personne et les autres. Elle est associée à l'entraînement collectif et contribue à son efficacité. D'autre part, le pratiquant s'exprime par des comportements «spontanés » non-contrôlés liés à des motivations inconscientes : la subjectivité de chaque personne est ici impliquée.

Alors que le maître guide la libération et énumère précisément les émotions à exprimer, lorsqu'il est nécessaire de contrôler les émotions, le patient est responsabilisé et n'est pas dirigé dans sa pratique. Les moyens de contrôle ne sont pas visibles ; seule la finalité compte.

L'expression des émotions doit donc être envisagée simultanément selon une double dimension sociale et individuelle. P. Dumouchel explique ce double aspect comme un attribut du corps : les émotions sont «le corps dans ce qu'il a d'indissociable de l'identité personnelle. Le corps n'est pas seulement fermeture sur soi, il est aussi l'inéluctable irruption du moi dans l'univers d'autrui ». Les émotions sont sociales : «avoir un corps, c'est être en communication, et cela est partie intégrante de l'expérience des émotions. La sociabilité des émotions est le cœur des phénomènes affectifs. Les émotions sont des œuvres communes auxquelles plusieurs participent ${ }^{(15)}$.

Selon R. I. Levy, « un comportement qui exprime des émotions est simultanément un symptôme... et une communication avec les observateurs ${ }^{(16)}$.

Dans sa définition, la composante socialement construite des émotions est déterminante : «Les émotions sont des sensations ou sentiments ${ }^{(17)}$ connectés aux relations extérieures au moi. Et ce moi... est intimement construit à partir de processus groupaux et de relations interpersonnelles ${ }^{(18)}$.

La faculté d'adopter deux types de conduites a priori opposées montre au bout du compte chez les pratiquants de qigong une capacité de maittrise de soi, d'adaptation de l'expression des émotions par la même personne selon le contexte et la motivation. Lattitude du maître diffère de celle des patients : ses émotions sont constamment contrôlées dans la pratique personnelle comme dans celle de soignant car il guide les comportements de l'ensemble du groupe.

\section{Qigong et états de transe : expression subjective et sociale des émotions, contrôle ou libération des émotions ${ }^{(19)}$}

J. Cosnier envisage les émotions "dans une acception étendue à tous les événements ou états du champ affectif qui se caractérisent par un ensemble d'éprouvés" psychiques spécifiques accompagnés, de façon variable en intensité et en qualité, de manifestations physiologiques et comportementales $\gg{ }^{(20)}$. La transe telle que la définit $\mathrm{G}$. Rouget peut être considérée comme un mode d'expression des émotions : « un état de conscience qui a deux composants, l'un psychophysiologique, l'autre culturel. Luniversalité de la transe signifie qu'elle correspond à une disposition psychophysiologique innée de la nature humaine, plus ou moins développée... suivant les individus. La variabilité de ses manifestations résulte de la diversité des cultures à travers lesquelles elle est mise en forme » ${ }^{(21)}$.

L'état de la personne généré par la pratique de qigong, se situe entre la veille et le sommeil banshui banxing: «Utiliser la pensée, ça a un rapport avec l'état de sommeil ; c'est entre l'état de veille et l'état de sommeil » ${ }^{(22)}$. La terminologie en usage indique un " état qui conduit au Vide de la pensée " ${ }^{(23)}$ : la référence à la notion de Vide est un héritage de la tradition; atteindre cet état est le 
but ultime de toutes les pratiques de méditation de haut niveau réservées aux initiés et transmises par les maîtres taoïstes, les moines bouddhistes, les lettrés confucéens, les maîtres d'arts martiaux et les praticiens de médecine chinoise traditionnelle. Cet objectif est un idéal commun aux pratiquants de toutes les écoles de qigong. L'étude de K. Miura s'appuie sur les biographies et les ouvrages des premiers maîtres de qigong (première moitié du XXe siècle) ; les discours contemporains d'un certain nombre de pratiquants, maîtres ou adeptes, sont extraits de mes données ethnographiques. Dans ces deux catégories de sources, la description des expériences vécues dans cet état se déroule selon les séquences suivantes :

- lorsqu'on entre dans cet état, une lumière blanche avec une tâche colorée de la taille d'un bouton apparaît devant les yeux;

- quand on est dans cet état, les points lumineux s'estompent, et on éprouve la sensation que le corps disparaît;

- la capacité de contrôle sur soi augmente ; la pratique peut être prolongée et s'accompagne d'une sensation de bien-être ;

- la résistance de la peau à l'électricité est réduite ${ }^{(24)}$.

Ces discours corroborent l'hypothèse que la pratique de qigong favorise la production d'états modifiés de conscience: A. M. Ludwig définit un état modifié de conscience comme " un état mental... représentant une déviation dans l'expérience subjective ou dans le fonctionnement psychologique par rapport à certaines normes générales de la conscience à l'état de veille " ${ }^{(25)}$.

Selon l'expression de G. Lapassade, les états modifiés de conscience sont des «transes à l'état potentiel» : "lls deviennent des transes effectives lorsque telle société choisit de "cultiver" tel ou tel de ces états " ${ }^{(26)}$.

L'étude des rapports sociaux, des systèmes de croyances et des rituels montre comment un état modifié de conscience se transforme en un phénomène de transe ${ }^{(27)}$. L'ensemble des processus à l'œuvre dans cet état peut être représenté sous la forme d'un continuum à polarités plutôt que sous la forme d'une opposition entre les deux polarités «transe et extase ». Le schéma ci-dessous ajoute des caractéristiques des pratiques du qigong aux deux séries d'opposition mises en évidence par G. Rouget indiquées dans la partie « modalités ».

Le pôle «transe " correspond à la pratique collective du qigong utilisée comme technique de soins alors que le pôle « extase » s'applique à la pratique corporelle individuelle visant à la préservation de l'état de santé. Les modalités comportementales du pôle « transe " tendent à libérer les émotions alors que celles du pôle « extase » conduisent à contrôler les émotions. Le tableau ci-dessus indique des tendances utiles pour décrire le processous mais les conduites et la perception réelles se situent du côté des états intermédiaires. Deux catégories de signes permettent de reconnaître l'état de transe : les symptômes sont « les signes qui ne constituent que l'expression brute et non élaborée d'une certaine perturbation vécue par le sujet au niveau de l'animalité ». Les conduites sont « les signes qui constituent non plus seulement une réaction... mais une action, chargée de valeur symbolique. Ils symbolisent toujours l'intensification d'une faculté ou d'une autre par le jeu d'actions revêtant un aspect extraordinaire ou étonnant ${ }^{(28)}$.

\begin{tabular}{|c|c|c|}
\hline & Transe & Extase \\
\hline Formes & $\begin{array}{l}\text { Collective } \\
\text { Passive }\end{array}$ & $\begin{array}{l}\text { Individuelle } \\
\text { Active }\end{array}$ \\
\hline Objectifs & Technique de soins & Pratique corporelle, préservation de la santé \\
\hline $\begin{array}{l}\text { Modalités } \\
\text { (extrait du } \\
\text { tableau de } \\
\text { G. Rouget) }\end{array}$ & $\begin{array}{l}\text { Mouvement } \\
\text { Bruit } \\
\text { Société } \\
\text { Avec crise } \\
\text { Surstimulation sensorielle } \\
\text { Amnésie } \\
\text { Pas d'hallucination }\end{array}$ & $\begin{array}{l}\text { Immobilité } \\
\text { Silence } \\
\text { Solitude } \\
\text { Sans crise } \\
\text { Privation sensorielle } \\
\text { Souvenir } \\
\text { Hallucination }\end{array}$ \\
\hline
\end{tabular}


Les pratiquants de qigong se trouvent dans l'état de transe selon les comportements décrits par G. Rouget à certains moments de la pratique: «La personne n'est pas dans son état habituel ; sa relation avec le monde qui l'entoure est perturbée; elle est en proie à certains troubles neurophysiologiques ; ses facultés sont - réellement, imaginairement ou fallacieusement - accrues ; cet accroissement se manifeste par des actions ou des conduites observables du dehors ${ }^{(29)}$.

Les mouvements involontaires du corps générés par la pratique sont-ils une expression des émotions ? Une expérience typique de conduites qui mettent en jeu des mouvements involontaires du corps, est décrite dans un ouvrage publié au début du siècle « Méthode de méditation de Maître Yinshi ${ }^{(30)}$, considéré dans le milieu des pratiquants de qigong comme un exemple de méthode évoquant, de manière précise, les comportements à adopter et les sensations à éprouver pour une pratique efficace. Lauteur Jiang Weiqiao est l'un des grands maîtres précurseurs du mouvement actuel. Il est devenu thérapeute en se guérissant lui-même par la méditation de la tuberculose et d'une hémorragie pulmonaire; il décrit cette expérience d'autoguérison dans le même ouvrage. Liu Guizhen en cite quelques extraits : «Tout à coup, il y eut un tremblement intense dans la partie basse de mon abdomen. J'étais assis en position de méditation, calme comme d'habitude, mais cette fois, quelque chose arrivait, que je ne pouvais vraiment pas contrôler. Ça me remuait d'avant en arrière sans recours. Ensuite, une énergie incroyablement chaude commença à croître à la racine de la colonne vertébrale, grimpa, de plus en plus haut, jusqu'à atteindre le sommet du crâne " ${ }^{(31)}$.

Le texte mentionne la répétition de l'expérience qui aboutit un jour à une véritable incorporation de ce $q i$ très chaud qui fera partie intégrante de lui-même : le $q i$ monte le long de la colonne jusqu'à la tête puis retourne par le visage et la poitrine jusqu'au bas de l'abdomen. Il décrit le circuit du microcosme (xiao zhou tian). Ainsi, il expérimente son corps comme le « corps-microcosme " décrit dans les traités taoïstes d'alchimie interne (neidan). L'énergie très chaude est connue, dans la tradition, comme l'énergie du ciel primordial, l'énergie interne. Les mouvements et les comportements observés chez les personnes qui sont plongées dans l'état de qigong sont en cohérence avec les représentations de la médecine chinoise traditionnelle et son modèle explicatif ${ }^{(32)}$. En effet, les types de comportements qui se manifestent sous l'influence du $q i$, correspondent à des types de pathologies affectant les Cinq viscères (wuzang) $)^{(33)}$ et les Six organes $(\text { liufu })^{(34)}$. Le principe du système de correspondances sous-tend les liens entre les Sept émotions et les "Cinq viscères / Six organes » : les patients qui ont tendance à être affectés par le rire en état de qigong sont atteints de maladies cardiaques ; ceux qui sont affectés par les pleurs ont une pathologie du foie, etc. ${ }^{(35)}$

Le vocabulaire des émotions donne des clés pour comprendre les représentations du corps et de la personne d'un point de vue distinctif. Cependant, il serait imprudent de surévaluer sa signification pour construire une théorie unifiée des émotions : récemment, les spécialistes ont remis en question le fait que les émotions constituent une classe d'objets homogènes ${ }^{(36)}$. Par exemple, le terme chinois qing signifie d'une part, " affection, sentiment, désir, amour », sens qui entrent dans la catégorie «émotion », et, d'autre part, « circonstances, fait réel, situation, vérité, raison, origine " sens qui réfèrent alors à plusieurs autres classes d'objets. Ainsi, selon Hansen Chad, « si les penseurs chinois partageaient les idées de la pyschologie populaire occidentale, alors les émotions et les sentiments devraient être des paradigmes de phénomènes intérieurs et subjectifs, l'exact opposé sémantique de "circonstances, fait et vérité" considérés comme extérieurs et objectifs " ${ }^{(37)}$. Pour P. Dumouchel, « le langage quotidien sur les émotions ne forme pas un tout cohérent dont on puisse tirer une théorie unifiée. Une théorie des émotions doit se construire en partie contre ce vocabulaire tout en rendant compte des intuitions au sujet des émotions qu'il éclaire et de la façon dont le langage des émotions est constitutif des émotions ${ }^{(38)}$. Peut-on donner une définition des émotions qui dépasserait la relativité culturelle et qui ferait consensus ? Cette question est l'objet d'un débat controversé fondé sur des arguments d'ordre éthique et scientifique ${ }^{(39)}$. Les émotions sont structurellement des états mentaux à composante physiologique et des productions sociales qui façonnent leur configuration. Jusqu'à quel point la culture et la société donnent-elles forme aux expressions et aux représentations des émotions sans pour autant remettre en cause la définition générique qui contient les termes d' " état mental " et de " composante physiologique ", des catégories elles-mêmes marquées culturellement ? La question clé est que si les émotions ne constituent pas une classe d'objets homogènes, la comparaison interculturelle fondée sur le vocabulaire des émotions n'est plus pertinente. Dans 
l'exemple de la pratique de qigong, l'expression des émotions est modelée par la subjectivité de l'individu et le jeu des rapports sociaux à l'œuvre dans ce contexte thérapeutique. Le contrôle ou la libération des émotions relève d'une attitude consciente orientée vers une finalité précise. L'expression "raisonnée " des émotions - la libération ou le contrôle étant posé comme principe - est un moyen d'atteindre l'objectif ultime du développement de la personne. Sa concrétisation implique une transformation de la personnalité et l'attitude prescrite pour faire face aux états émotionnels est un élément transformant fondamental. Dans la pratique du qigong, la manipulation de l'expression des émotions est une technique thérapeutique fondamentale constamment utilisée par les «maîtressoignants".

Le qigong suscite un engouement croisant auprès des populations occidentales et figure en bonne position au sein des recours thérapeuthiques désignés sous le terme générique de «médecines douces, parallèles, alternatives ». Par exemple, le mouvement falungong dont le maitre fondateur réside actuellement aux EtatsUnis, compterait parmi ses adeptes environ 50 millions de personnes hors de Chine. De nombreuses publications portant sur le travail du $q i$ existent en langues occidentales; les associations de qigong se sont multipliées en Europe et en Amérique du Nord. De plus, parmi les recours parallèles, les pratiques du $q i$ présentent un intérêt spécifique par leur rapport avec les sciences biomédicales : c'est une médecine énergétique qui fait l'objet de recherches expérimentales à un niveau international ${ }^{(40)}$.

Le qigong est donc promis à un bel avenir, du moins à une longévité certaine, dans les mondes chinois et occidental. $\oplus$

F

1. Tuina désigne dans ce contexte non pas des massages selon le sens contemporain usuel mais l'ensemble des techniques de respiration. L'idée de "Rejeter tout ce qui est vieux pour assimiler tout ce qui est nouveau " apparaît dans le chapitre 15 du Zhuongzi, Taipei, Dongda dushu, 1988.

2. Yinian a un sens pratique et désigne l'action de penser, la pensée opératoire, la volonté, concept qui s'oppose à siwei la pensée abstraite, discursive.

3. Dejà dans le Huainanzi, ouvrage de philosophie politique datant du lle siècle avant $\mathrm{J}$. C., le contrôle des émotions apparaît comme un travail de régulation du qi et une condition nécessaire pour atteindre l'état de "sagesse ": "la différence entre des sages qui agissent de manière autonome [c'est-à-dire libérés de leurs émotions] et des "non-sages" qui ne le font pas, est présentée comme une différence dans la façon de réguler les mouvements du qi à l'intérieur de leurs corps", cf. G. Vankeerberghen, "Emotions and the Actions of the Sage: Recommendations for an Orderly Heart in the Huainanzi ", Philosophy East and West, vol. 45(4), 1995, pp. 527-544 (p. 540).

4. Cf. C. Despeux, "Le qigong, une expression de la modernité chinoise ", in J. Gernet et M. Kalinowski eds., En suivant la Voie Royale - Mélanges en hommage à L. Vandermeersch, Paris, EFEO, Etudes thématiques 7, 1997, pp. 267-281.

5. Pour une étude approfondie des pratiques de qigong, des formes d'organisations sociales et des publications en langue chinoise, $\mathrm{cf}$. E. Micollier, Un aspect de la pluralité thérapeutique en Chine populaire : les protiques de qigon -. Dimension thérapeutique/dimension sociale, Thèse de doctorat en Anthropologie, Aix en Provence, Université de Provence, 1995.

6. Chiffres donnés par Annual China Review, " The Call of the Past : qigong and Clan Revivalism ", Hong Kong, Hong Kong University Press, 1991 et China Review, décembre 1988, p. 39.

7. Cf. Asian Woll Street Journal, 26 avril 1999, Pp. 1-2.

8. Séquences de discours d'informateurs qui ne sont pas obligatoirement des pratiquants de qigong. L'analyse des données collectées est en cohérence avec les résultats de l'étude de $M$. P. Y. Tung (1994) qui a interrogé des personnes de culture chinoise vivant en Californie (Baie de San Francisco) ayant suivi une éducation secondaire République populaire de Chine ou à Taiwan, capables de lire et parler couramment le chinois mandarin, cf. M. P. Y. Tung "Symbolic Meanings of the Body in Chinese Culture and "Somatization", Culture, Medicine and Psychiatry vol. 18, $n^{\circ} 4$, 1994, pp. 483-492.

9. Selon la théorie basique de la médecine chinoise traditionnelle, l'influence des Sept émotions sur un étre humain varie en fonction des circonstances : 1-xi, joie, gaîté, excitation, 2-nü, colère, irritation, 3-you, anxiété, chagrin, 4-si, méditation, contemplation, inquiétude, 5-bei, affliction, peine (caractérisée par un son guttural sans larmes), 6-kong, peur, anxiété extrême, 7-jing, effroi, peur soudaine et intense, cf. F. Liu F. et Y. Liu, Zhongyi mingci hui bian (Terminologie de médecine chinoise), Hong Kong, The Commercial Press, 1980, p. 18. Les Sept émotions sont en correspondance avec les Cinq viscères wuzang : foie-colère $(n \ddot{)})$, cœur-joie $(x i)$, rate-inquiétude (si), rein-peur (kong) et effroi (jing), poumon-chagrin (you) et affliction (bei). D'une part, les émotions induisent ou aggravent la maladie, et génèrent des troubles somatiques : elles sont donc des facteurs pathogènes, cf. T. Ots, "The Angry Liver, the Anxious Heart and the Melancholy Spleen. The Phenomenology of Perception in Chinese Culture ", Culture, Medicine and Psychiatry, vol. $14 n^{\circ} 1,1990$, pp. 21-58. D'autre part, elles sont aussi des symptômes : une colère excessive est un symptôme du désordre du foie. Sur l'ambiguité entre facteur pathogène et symptôme, cf. C. Despeux, F. Obringer, "Conceptualisation d'un état pathologique dans la médecine chinoises traditionnelle : exemple de la toux $n$, Revue d'Histoire des Sciences, 1990, vol. XLII, n¹, pp. $35-$ 56 ; C. Despeux, F. Obringer eds., La maladie dans la Chine médiévale : lo toux, Paris, L'Harmattan, 1997.

10. Dictionnaire utilisé ici : Cowie A. P., Evison A., Zhu Yuan, Wang Liangbi, Wu Jingrong et Mei Ping, Concise English-Chinese, Chinese-English Dictionary, Jingxuan Han-Ying/Ving-Han Cidian, Hong Kong, Oxford University Press and The Commercial Press, 1986.

11. Cf. A. P. Cowie et al., op. cit., 1986.

12. $D^{\prime}$ autres termes servent à désigner la passion reqing et qinggan.

13. L'un des deux maîtres de qigong de Canton avec qui j'ai pu contruire un récit de vie.

14. Se mouvoir, faire des mouvements de manière désordonnée.

15. P. Dumouchel, Emotions. Essai sur le corps et le social, Synthélabo, coll. " Les Empécheurs de penser en rond ", Paris, 1995, pp. 1416.

16. Cf. R. I. Lew, "Emotion, Knowing and Culture ", in R.A. Schweder 
et R. Le Vine Culture theory : Essays on Mind, Self, and Emotion, Cambridge, Cambridge University Press, 1984, pp. 214-237 (p. 230).

17. Feelings en anglais.

18. Cf. R. I. Levy, op. cit., p. 221.

19. Cette partie est adaptée du texte de la thèse de doctorat de l'auteur, cf. E. Micollier, op. cit. 1995, pp. 192-196.

20. Cf. J. Cosnier, Psychologie des émotions et des sentiments, Paris, Retz, 1994

21. Cf. G. Rouget La musique et la transe, Paris, Gallimard, 1980, p. 39.

22. Discours récurrent parmi les maîtres de qigong.

23. Plus précisement, wu yishi tai : état de la " connaissance de l'absence de la pensée $n$.

24. Cf. K. Miura, "The Revival of Qi : Qigong in Contemporary China ", in L. Köhn ed., Taoist Meditation and Longevity Techniques, Ann Arbor, University of Michigan, 1989, Pp. 331-362.

25. Cf. A. M. Ludwig, "Altered States of Consciousness ", in R. Prince ed. Trance and Possession States, Montréal, 1968.

26. Cf. G. Lapassade, La transe, Paris, PUF, 1990, p. 9.

27. Ibid., p. 10.

28. Cf. G. Rouget, op. cit., pp. 56-57.

29. Ibid., p. 58.

30. Jiang Weiqiao, Yin Shizi jingzuofa (Méthode de méditation de Maître Yinshi), Taipei, 1967.

31. Liu Guizhen, Qigong jingxuan (Sélection de gigong), Pékin, Renmin tiyu chubanshe, 1981, p. 75.

32. Rappelons que le système conceptuel qui sous-tend cette médecine, est la théorie du système des correspondances, cf. P. U. Unschuld, Medicine in China. A History of Ideas, Berkeley, University of California Press, 1985, pp. 51-100 ; cf. S. Nathan, Traditional Medicine in Contemporary China, Ann Arbor, University of Michigan, 1987.

33. Cœur, foie, rate, poumons, reins. Appartenant à la phase yin, ces organes remplissent la fonction vitale de régénérer et de stocker le jingqi, le " qi essentiel de la vie ", cf. F. Liu et Y. Liu, op. cit., 1980, p. 26. Les viscères et les organes internes sont désignés par deux catégories : zang et fu. Les wu zang sont les organes internes situés dans les cavités abdominales ou thoraciques.

34. Vésicule biliaire, estomac, intestin grêle, gros intestin, vessie, et les "Trois Réchauffeurs " (san jiao), un groupe d'organes identifiés selon les trois positions dans lesquelles ces organes sont localisés et fonctionnent. Il s'agit 1) d'indiquer les qualités d'extériorité biao ou d'intériorité $l i$, telles qu'elles sont illustrées par les $f u$ et les zang respectivement ; 2 ) de montrer les relations physiologiques suivantes. Ainsi, le cœur est coordonné avec et complémentaire de l'intestin grêle; la rate avec l'estomac; le foie avec la vésicule biliaire ; les poumons avec le gros intestin; le péricarde avec les "Trois Réchauffeurs »., $f$. F. Liu et Y. Liu, op. cit., 1980, pp. 26-27.

35. Les catégories nosologiques de la médecine chinoise traditionnelle sont mises en correspondance avec les "Sept émotions " ( $q i$ qing).

36. Cf. R. Solomon, "Some notes on Emotions East and West ", Philosophy East and West, vol. 45, n², 1995, pp. 171-202; J. Marks et R. T. Ames eds., " Emotions in Asian Thought. A Dialogue in Comparative Philosophy ", Albany, State University of New York, 1995.

37. Cf. Hansen Chad, "Qing (Emotions) in Pre-Buddhist Chinese Thought ", in J. Marks et R. T. Ames eds., op. cit., pp. 181-211 (pp. 182-183).

38. Cf. P. Dumouchel, op. cit., p. 18.

39. Pour une synthèse sur ce débat, cf. R. Solomon, op. cit.

40. Cf. J. C. Crombez, "Le Qi-Gong au Canada, de l'étrange au familier, y a-t-il un chemin? ", Santé Culture. Culture, Health, vol. IX, $\mathrm{n}^{\circ} 2$, 1992-1993, pp. 281-301 ; E. Micollier, "Entre science et religion, modernité et tradition : le discours pluriel des pratiquants du qigong ", in Jean Benoist ed., Soigner au pluriel - Essais sur le pluralisme médical, Paris, Karthala, 1996, pp. 205-223.

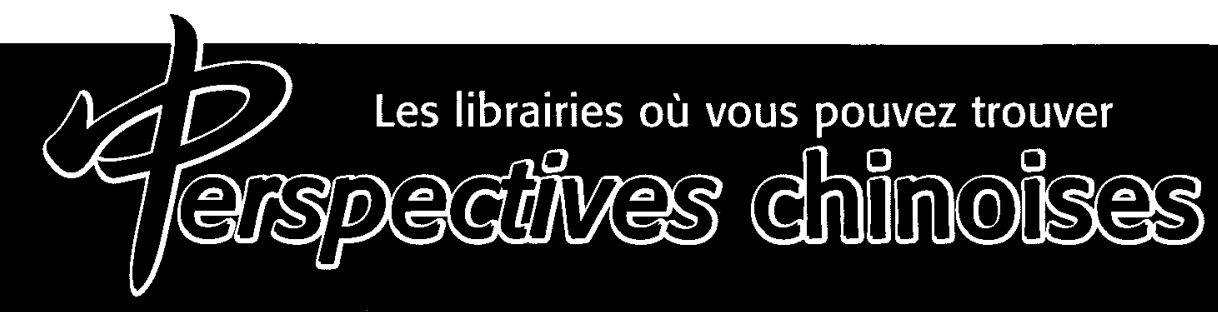

FRANCE

\section{Dunod Dauphine}

Hall de l'Université

Place du Maréchal de Lattre de Tassigny 75116 Paris

Itinéraitres

60, rue Saint Honoré 75001 Paris

\section{La Boutique de FHistoire}

24, rue des Ecoles

75005 Paris

\section{La Terrasse de Gutenber:}

9, rue Emilio Castelar

75012 Paris

\section{Librairie de la Documentation française}

31, quai Voltaire

75007 Paris

\section{Lbrairie du CFCE}

10 , avenue d'lena 75016 Paris

\section{Lbrairie Jonas}

14, rue Maison Blanche 75013 Paris

49, rue Gay-Lussac 75005 Paris

\section{Les FNAC}

Montparnasse, Les Halles,

Etoile, St-Lazare, Champs-Elysées

\section{LHarmattan}

16, rue des Ecoles 75005 Paris

\section{La Procure}

3 , rue de Mézières 75006 Paris

\section{Fençtre sur YAsie}

Le Phénix

72 , boulevard de Sébastopol

75003 Paris

Nouveautés sur le Web

http://perso.wanadoo.fr/librairie.lephenix

Lbrairie des sciences polfitiques 30, rue St Guillaume 75007 Paris

You Feng

45, rue Monsieur le Prince 75006 Paris

\section{HONG KONG}

\section{Parenthèses}

4/F, Duke of Wellington House 14 Wellington Street, Central

\section{TAIWAN}

\section{Estite}

2/F, 245 Tun-Hwa South Road, Taipei

\section{Caves books}

103 Chung-shan North Road, Sec. 2, Taipei 